\title{
L'ESPAGNE DU XIX SIÈCLE VUE PAR UN HUSSARD: JOHN ROCCA, UN PRÉCURSEUR DES VOYAGEURS ROMANTIQUES FRANÇAIS
}

\author{
MontserRat SERRAno MaÑES \\ Universidad de Granada \\ mserrano@ugr.es
}

\section{RESUMEN}

Si existe un momento histórico en el que España se convierte en un espacio privilegiado en la producción literaria francesa, ese período es el siglo XIX: es entonces cuando se asocian estrechamente tópicos y color local, elementos tan apreciados por los Románticos. Pero antes de la oleada de viajeros de los años 1820-1830, ha habido una primera oleada de hombres que ha conocido España arrastrada por las campañas napoleónicas. Será a través de los relatos de los militares como se transmitirá, al menos parcialmente, la imagen fundadora de la España romántica. Si la mayor parte de estos relatos son guerreros, los hay que revelan un empeño literario claro. Es el caso de las Mémoires sur la guerre des français en Espagne, de John Rocca, precursor de la moda de la literatura de viajes, y en especial del viaje a España. Obra en la que convergen el relato de viaje, el nacimiento y germen de una moda literaria, la comprensión del relato de guerra y de los lugares de memoria. Estas Memorias son no solo una simple descripción del relato de guerra, sino también una obra literaria.

PALABRAS ClaVE: Relato de guerra, literatura de viajes, precursor, España.

\section{RÉSUMÉ}

S'il y a un moment historique où l'Espagne devient un espace privilégié dans la production littéraire française, cette période est le XIX siècle: c'est alors que l'on associe 
étroitement topiques et couleur locale, éléments tant appréciés par les Romantiques. Mais avant les voyageurs des années 1820-1830, une première vague d'hommes, entraînée par les guerres napoléoniennes, a connu l'Espagne. Ce sera à travers les récits des militaires que se transmettra, en partie au moins, l'image fondatrice de l'Espagne romantique. Si la majeure partie de ces récits sont des relations guerrières, il en est qui révèlent des soucis littéraires clairs. C'est le cas des Mémoires sur la guerre des français en Espagne, de John Rocca, précurseur de la mode de la littérature de voyages, et notamment du voyage en Espagne. Un ouvrage dans lequel convergent le récit de voyage, la naissance et le germe d'une mode littéraire, la compréhension du récit de guerre et des lieux de mémoire. Ces Mémoires sont non seulement une simple description de récit de guerre, mais aussi une œuvre littéraire.

MoTs CLÉs: Récit de guerre, littérature de voyage, précurseur, Espagne.

L'imagologie culturelle et littéraire transmise par les visiteurs de la Péninsule depuis le Moyen Âge, véhicule, dans une majorité des cas, la culture et les opinions de la «culture regardante», selon l'expression de D.-H. Pageaux (1995:137). La représentation de l'étranger étant toujours passée par le philtre de la culture et des mœurs du pays duquel procède le visiteur, le récit de voyage aura toujours, de par ce fait, un caractère exogène incontournable. L'image transmise par ces écrits maintient forcément des rapports avec le moment historique et culturel de production du document, auquel vient s'ajouter dans tous les cas un décalage plus ou moins grand, dû à l'écart entre le voyage et la phase d'écriture de son récit.

S'il y a un moment historique où l'Espagne devient un espace privilégié dans la production littéraire française, cette période est bien le XIX siècle: c'est alors que l'on associe étroitement topiques et couleur locale, éléments tant appréciés par les romantiques. On récupère l'image mythique d'un temps, passé par le filtre de l'imagination. Le voyage permettra à beaucoup d'auteurs de créer et de recréer une imagologie favorisée par l'exterritorialité dans laquelle ils se retrouvent immergés en traversant les frontières.

Cependant, avant les voyageurs des années 1820-1830, une première vague d'hommes, entraînée par les guerres napoléoniennes, a connu l'Espagne. Ce sera à travers les récits des militaires - simples soldats, hussards ou généraux - que se transmettra, en partie au moins, l'image fondatrice de l'Espagne romantique ${ }^{1}$. Certes, la majeure partie de ces récits sont des relations guerrières, ce qui vient confirmer la fluctuation terminologique et le caractère hybride du genre viatique. Souvent, bien des années se sont écoulées entre le moment des faits, c'est-à-dire le voyage, et leur publication, et ces narrations mettent tout simplement en action le conteur, qui se pose volontiers en héros en faisant la relation des faits, ou qui par ses descriptions et ses commentaires explique à des éventuels lecteurs ses réactions face à des réalités qui lui ont été étrangères.

1 «Pour de multiples raisons, l'Espagne est à la mode au cours du premier tiers du XIX siècle. Des milliers de Français lancés dans la lamentable invasion napoléonienne de 1808 ont acquis une connaissance du pays voisin autrement approfondie que celle de Gustave Flaubert» (Aymes, 1983: 8). 
Mais il en est qui révèlent des soucis littéraires évidents, et qui ont été offerts au public avec un décalage temporel assez court. C'est le cas du texte qui nous occupe : les Mémoires sur la guerre des français en Espagne, de John Rocca, publiées en 1814, et qui ont pour sujet les campagnes napoléoniennes sur le sol ibérique entre la fin de l'année 1809 et juillet 1810. Face aux lectures et aux approches des voyages en Espagne d'auteurs reconnus et réputés tels que Laborde, Mérimée, Gautier, les mémoires de Rocca, témoin en première ligne de combat des guerres napoleóniennes sur le sol espagnol, sont, de par leur qualité littéraire et de par leur contenu, une espèce de pierre de touche pour la compréhension de tout un imaginaire exterritorial; dans son ouvrage, trop méconnu de nos jours, convergent le récit de voyage, la naissance et le germe d'une mode littéraire, la compréhension du récit de guerre et des lieux de mémoire ${ }^{2}$.

Mais avant de passer à l'approche de son œuvre, quelques touches sur sa biographie s'avèrent nécessaires. Nous lèverons ainsi ne serait-ce que légèrement, le voile qui cache depuis si longtemps cet écrivain oublié. Albert Jean Michel Rocca, connu à Genève, sa ville natale, sous le nom de John Rocca, naît en 1787 ou 1788 au sein d'une famille de notables. Après son adolescence, il fait ses études à École Polytechnique de Paris, pour suivre sa vocation militaire. Sous-lieutenant des hussards, il participe aux campagnes de la Prusse et de l'Espagne. Grièvement blessé, près de Ronda, il rentre en France en 1811. C'est à Paris qu'il rencontre Madame de Staël. Devenus amants, ils voyagent ensemble à partir de 1812, et se marient dans le secret en 1816. De leur liaison naîtra clandestinement un fils. Leur retour à Paris sera de courte durée: le retour de l'Empereur les oblige à un nouvel exil à Coppet. Tous les deux ont déjà, à ce stade de leur vie, une santé très précaire. Madame de Staël, en fine critique, pousse John Rocca à raconter ses souvenirs de guerre, dans lesquels, apparemment, elle a participé pour alléger ou corriger. La phtisie emporte Rocca en 1818, quelques mois seulement après la mort de son épouse Madame de Staël.

Les Mémoires sur la guerre des Français en Espagne a remporté immédiatement un notable succès, et furent accueillis avec des éloges, malgré quelques réticences de part et d'autre des Pyrénées: les Français considérèrent l'ouvrage trop favorable à l'Espagne, tandis que les Espagnols, de leur côté, critiquèrent son excessive glorification de l'Armée française. Mais l'oeuvre fut plusieurs fois rééditée et traduite, aussi bien en France qu'en Angleterre, où les Mémoires paraissent en anglais en 1815; en Italie, qui connaît une traduction en 1816, ou en Allemagne, où l'on traduit l'ouvrage en 1908. En 1855, M. C. R. de Croy publie Épisodes de voyages, dans lequel on inclut un fragment de l'œuvre de Rocca: le récit de son départ pour Séville - ville qu'il n'atteindra pas - après avoir traversé Andújar, Córdoba, Écija et Carmona, et son passage par Ronda avec son régiment, jusqu'au moment où, assez récupéré de sa blessure pour reprendre son chemin, il quitte cette ville où il a été remarquablement soigné, et rentre en France. Il faut dire que l'œuvre est traduite à l'espagnol en 1816 par Angel Arenal, et qu'elle sera reprise quelque temps plus tard avec des variantes, dans «La Ilustración Católica». Son succès

${ }^{2}$ B. Bennassar (1998: VII) signale précisément les qualités littéraires, historiques et humaines de ces récits de militaires trop oubliés: «si quelques-uns de ces soldats ne savent raconter que «leur» guerre et les atrocités des guerrilleros en oubliant pieusement les forfaits de leur propre armée, il en fut d'autres qui découvrirent un autre pays». 
s'est prolongé jusqu'au début du XX siècle, car elle a été traduite de nouveau par A. Salcedo en 1908.

John Rocca réalisera deux séjours guerriers dans la Péninsule. Si dans ses premières campagnes il parcourt le Nord de l'Espagne, Madrid et l'Extremadura notamment, où il participe à la bataille de Medellín, en 1809 ses campagnes le mènent enfin jusqu'en Andalousie. C'est déjà, pour le préromantique Rocca, la région qui embrasse tous les traits et caractères topiques qui fonderont pendant longtemps la vision de l'Andalousie, et avec elle celle de l'Espagne ${ }^{3}$.

Lors de sa première campagne surtout, Rocca fait abondamment état des faits d'armes: les mouvements des troupes, leurs victoires successives sont détaillés de manière précise. Les touches descriptives font nonobstant foi de ses visées non seulement militaires, mais littéraires. Les paysages et les villes désertées par leurs habitants profitent du regard aux relents romantiques de l'auteur:

En approchant des villes et des bourgades désertes de la Castille, on ne voyait plus ces vapeurs de fumée qui, s'élevant sans cesse dans les airs, forment une seconde atmosphère au dessus des cités habitées et populeuses. Au lieu des bruits vivans, des rumeurs continuelles, on n'entendait dans l'enceinte des murs que les heures mortes, dont notre arrivée n'avait pu suspendre le cours; ou bien les croassemens des corneilles qui planaient autour des clochers élevés. Les maisons, vides pour la plupart, n'étaient plus que des échos qui répétaient d'une manière tardive et en désaccord les sons éclatants des tambours et des trompettes (Rocca, 1887: 24).

Mais le spectacle de Madrid, ville grouillante de monde, avec ses différences et l'aspect bariolé de ses gens, frappe aussi le conteur, qui se laisse aller, après les longs moments de relation des mouvements des troupes, à décrire dans la suite du récit des aspects, des mœurs - la sieste l'étonne déjà dans son long séjour dans la capitale (Rocca, 1887:56) - et des lieux singuliers pour lui, en tant qu'observateur étranger. Ses commentaires explicatifs adressés au lecteur - «Vous voyez», «vous rencontrez» (Rocca, 1887: 55) - mettent en relief ses réactions dans la confrontation avec une réalité allochtone. Ce regard sur l'hétérogène s'appuie bientôt sur le motif de la couleur locale. De ce long fragment, dans lequel Rocca s'épanche sur cette vision de l'Autre, il est à signaler comment il singularise déjà, parmi tous ces êtres, le type de l'Andalou, à peine encore dessiné. Il annonce dès lors la place majeure accordée à l'Andalousie dans son récit, comme elle le sera dans les narrations des «maitres en voyage» qui le suivront à partir des années $1820^{4}$ :

On est étonné en entrant dans Madrid le matin par la porte de Tolède et la place de la Cevada, où se tient le marché, du concours tumultueux de gens de la campagne et des provinces,

3 C'est à propos de la valorisation du côté exotique que Maria del Mar Serrano (1993:14) fait cette pertinente remarque: «El interés generalizado de los viajeros extranjeros por España comenzó en el siglo XIX, en parte porque la guerra mantenida contra los franceses desde 1808 a 1813, en la cual se involucró también Inglaterra, paradójicamente propició el conocimiento de la Península en el resto d Europa pero, sobre todo, porque la finalidad de los viajes había cambiado ya para entonces».

${ }^{4}$ D'après René Aymes (1983:16), «La véritable Espagne, selon le critère des voyageurs romantiques, est donc andalouse, c'est-à-dire semi-africaine. D'où l'exclusion, totale ou partielle, des trois quarts du territoire national». 
diversement vêtus, qui arrivent, partent, vont et viennent. Là un Castillan relève avec dignité les plis de son large manteau, comme un sénateur romain enveloppé dans sa toge. Ici un bouvier de la Manche, un long aiguillon à la main, est revêtu d'une saie de peau de buffle, qui ressemble encore, par sa forme ancienne, à la tunique que portaient les guerriers romains et les Goths. Plus loin on voit des hommes dont les cheveux sont enveloppés dans de longs réseaux de soie; d'autres portent une espèce de veste courte, brune, chamarrée de bleu et de rouge, et qui rappelle le vêtement mauresque. Les hommes qui portent ce vêtement viennent de l'Andalousie; on les reconnaît à leurs yeux vifs et noirs, à des regards plus expressifs et plus animés, à un langage plus rapide (Rocca, 1887:54).

Le fait d'être un soldat français ne lui fait perdre en aucun instant un point de vue objectif face à ce peuple qui se rebelle devant l'envahisseur, qui se relève vivement après chaque défaite, malgré l'indiscipline ou le manque de chefs. Ses digressions montrent non pas de la haine, mais bien plutôt une certaine admiration pour le caractère espagnol, dont il souligne à maintes reprises les qualités, en remarquant le bien-fondé de certains comportements: «Leur animosité s'accroissait de toutes les vexations que les Français leur faisaient éprouver» (Rocca, 1887:142). Il loue, par exemple, la sobriété des Andalous et des Espagnols, qualité qu'il considère un effet positif de l'esprit religieux qui présiderait à la vie des hommes de la Péninsule, ainsi que du souvenir des ancêtres Arabes. L'image mythique des ascendants arabo-musulmans est déjà mise en parole, à partir de cette digression, par Rocca:

Les Andalous et les Espagnols, en général, sont sobres comme les orientaux, au milieu même de l'abondance, par un principe religieux; ils regardent l'intempérance comme un abus des dons que Dieu accorde, et méprisent profondément ceux qui s'y livrent (Rocca, 1887:167).

Ailleurs, ses considérations sur la situation du roi Joseph en Espagne, et sur le peu de faveur qu'il compte parmi le peuple espagnol, permettent d'entrevoir en même temps ses propres opinions, à travers le choix discursif. Le substrat comique de l'anecdote insérée, dont les temps verbaux soulignent l'itération, laisse apparaître dans un phénomène de transparence textuelle ses propres idées:

Les dévots, qui étaient habitués à mêler dans leurs discours cette exclamation, Jésus, $M a$ rie, Joseph, s'arrêtaient court dès qu'ils avaient prononcé les deux premiers de ces trois mots, et après une pause, ils se servaient de cette périphrase, et le père de notre Seigneur, craignant d'attirer une bénédiction sur le roi Joseph, en nommant le saint qui était censé être son patron dans le ciel (Rocca, 1887:117).

L'itinéraire de sa traversé de la Péninsule, au fil des étapes, semble brouiller l'itinéraire référentiel dans une «traversée de l'écriture» parallèle. Car bien que le récit soit ancré dans le réel, les pistes de ce qui peut être considéré aussi comme un récit de voyage s'enchevêtrent dans des références temporelles et historiques qui estompent les limites entre réalité vécue et connaissances acquises. Son entrée en Andalousie est un point marquant du récit, signalé comme l'arrivée dans un nouveau monde:

Dès qu'on arrive sur le revers des montagnes on est en Andalousie; on éprouve alors une différence sensible dans la chaleur de l'atmosphère, et la magnificence des campagnes qu'on 
découvre devant soi forme un grand contraste avec la stérilité de la montagne noire ou SierraMorena d'où l'on sort. Les cultivateurs étaient occupés à la récolte des olives, et la campagne avait, vers la fin de l'hiver, cet aspect riant et animé qu'on ne remarque dans les autres pays plus au nord, que pendant le temps de la moisson et des vendanges (Rocca, 1887:158).

Ses descriptions se conforment souvent avec les normes littéraires, et c'est surtout à travers des descriptions statiques, ponctuées de verbes du type «voir» — «On voyait», «On aperçoit» (Rocca, 1887:159) - que le narrateur se porte garant de la vérité de ses dires. On pourrait même saisir une dimension intertextuelle si l'on rapproche sa peinture invraisemblable des montagnes andalouses, avec au loin une vision physiquement impossible de Sierra Nevada, de celle postérieure et non moins invraisemblable du maitre romantique Gautier dans son Voyage en Espagne ${ }^{5}$, le génie littéraire romantique de ce dernier en moins:

À notre gauche étaient les montagnes du royaume de Jaen, et l'on distinguait dans l'éloignement les sommets toujours couverts de neige de la Sierra-Nevada de Grenade. Ces cimes ont été les derniers asiles où les Maures se sont retirés avant leur dernière expulsion de l'Espagne (Rocca, 1887:159).

Rocca rassemble de la sorte un bon nombre de topoi qui appartiennent en propre à l'écriture viatique. Il insère descriptions et digressions plus ou moins longues qui rapportent le lecteur vers un passé mythique: le topique du Maure se met déjà en place. A côté de cela, son souci littéraire le porte à arranger des passages descriptifs jouant avec les temps verbaux. Une certaine complexité dans la construction qui ne fait que souligner ses visées esthétiques et qui met en relief les deux plans énonciatifs. Le narré est bâti fondamentalement sur l'imparfait remémoratif : «passait», «voyait», «était». La linéarité temporelle du passé se trouve alors perturbée par l'inclusion d'un nombre considérable de présents de l'indicatif — «croissent», «sont bordés», «sont», «s'élèvent», «on aperçoit», «conservent»-, ce qui constitue une actualisation narrative où le présent de l'énonciation semble rattraper l'horizon du passé:

La route passait entre de longues plantations d'oliviers à l'ombre protectrice desquels croissent alternativement le blé et la vigne. Les champs sont bordés de haies d'aloès dont les feuilles sont aiguës comme des lances, et dont les tiges menues s'élèvent perpendiculairement aussi haut que des arbres. On voyait de distance en distance, derrière les habitations, des vergers touffus plantés d'orangers, et, dans les terrains incultes sur les bords des ruisseaux, des lauriers roses et blancs qui étaient alors en fleurs. On aperçoit quelquefois encore, de loin en loin, de vieux palmiers que les curés conservent dans leurs jardins pour en distribuer les branches à la fête des rameaux (Rocca, 1887:159).

5 «La ville de Bailén, avec ses toits de tuiles, son église rouge et ses maisons blanches accroupies au pied de la tour comme un troupeau de chèvres, formait un admirable premier plan; plus loin, les champs de blé ondoyaient en vagues d'or, et tout au fond, au-dessus de plusieurs rangs de montagnes, l'on voyait briller, comme une découpure d'argent, la crête lointaine de la Sierra Nevada» (Gautier, 1981:248). Gautier réalise son voyage en Espagne en 1840, et il se peut qu'il ait lu d'abord le récit, à l'époque assez connu, de John Rocca. Chez Gautier, la description de voyage devient œuvre littéraire à par entière, mais que ce soit Bailén ou Santa Cruz, les lois de la physique empêchent que l'on puisse voir, de ces endroits-là, la Sierra Nevada. 
La marche militaire conduit le narrateur le long du Guadalquivir vers Séville. Les descriptions physiques se muent alors en descriptions qui se veulent anthropologiques. Ce regard sur l'hétérogène fixe son discours sur l'autre à travers des caractères et des caractéristiques topiques. Dans un souci évident d'intéresser le lecteur, Rocca insère des anecdotes, quelque proverbe ${ }^{6}$, ou des digressions, expliquant par exemple pourquoi les Andalous mangent tous les jours du porc ${ }^{7}$. Et l'imagologie portant sur cet ailleurs lointain offre une image immuable de l'Andalousie, «sans contredit, la contrée de l'Espagne la plus fertile et la plus opulente par la nature» (Rocca, 1887:160); le souvenir de son passé arabe devient fondement scriptural et repère incontournable. Ses caractéristiques référentielles embrassent d'ailleurs toute l'Espagne:

On rencontre presqu'à chaque pas, en Andalousie, plus encore que dans les autres provinces de la péninsule, des traces ou des souvenirs des Arabes, et c'est ce mélange singulier des coutumes et des usages de l'orient avec les mœurs chrétiennes, qui distingue particulièrement les Espagnols des autres peuples de l'Europe.

Les maisons des villes sont presque toutes construites à la mauresque; elles ont dans l'intérieur une cour pavée avec des grandes dalles en pierres plates, au milieu de laquelle est un bassin d'où jaillissent des jets d'eau qui rafraîchissent sans cesse l'air; ce bassin est ombragé par des citronniers ou des cyprès. Des treilles d'orangers sont quelquefois adossés aux murs, et ces arbres portent pendant toute l'année des feuilles, des fleurs ou des fruits (Rocca, 1887:161).

Son passage par Cordoue est peut-être un texte inaugural, par sa description de la mosquée qu'il décrit avec minutie. Les touches anthropologiques renvoyant à une réalité qui se veut plus large ${ }^{8}$ - quoique incertaine pour de possibles lecteurs espagnols - côtoient les élancements lyriques soulevés par la magnificence du monument: «Cette multitude de colonnes, surmontées d'arcades, rappelle une immense forêt de palmiers, dont les rameaux, régulièrement recourbés, se toucheraient en s'inclinant» (Rocca, 1887:163). Par la suite, Cordoue avec ses rues silencieuses et sa mosquée seront incontournables pour tout voyageur. Mais Rocca donne déjà, dans ces Mémoires, le ton. Les pages sur la capitale andalouse, focalisées sur la mosquée, embrassent et le monument et ses connotations religieuses, et ses appréciations deviendront par la suite un lieu commun:

Il y a une de ces cours à découvert dans l'enceinte même de la cathédrale de Cordoue, qui est une ancienne mosquée. Cette cour, comme celle des maisons des particuliers, est ombragée par des citronniers et des cyprès, et elle renferme des bassins où l'eau se renouvelle sans cesse par des jets perpendiculaires. Lorsqu'on entre dans la partie consacrée de la mezquita ou mosquée, c'est le nom que ce temple conserve encore de nos jours, on est frappé d'étonnement

\footnotetext{
6 «Il y a un proverbe dans les Castilles et dans la Manche, qui dit que l'eau seule du Guadalquivir engraisse plus les chevaux que l'orge des autres pays» (Rocca, 1887:160).

7 «Cette viande, malsaine dans les pays chauds, est prohibée par la loi sainte de tous les peuples de l'orient, et elle leur est en horreur. Dans le temps où l'Espagne fut reconquise par les chrétiens sur les Maures, avant l'entière expulsion de ces peuples, il y avait en Andalousie beaucoup de musulmans et de juifs qui s'étaient convertis en apparence seulement, pour avoir la permission de rester dans le pays; les Espagnols chrétiens mangeaient alors du porc pour se reconnaître entre eux, et c'était, si l'on peut s'exprimer ainsi, une espèce de profession de foi» (Rocca, 1887:167).

8 «Ces sortes de rapprochements se rencontrent sans cesse en Espagne, et rappellent le triomphe du christianisme sur l'islamisme» (Rocca, 1887:163).
} 
à la vue d'une multiplicité de colonnes de marbre de diverses couleurs. Ces colonnes sont rangées en allées parallèles assez rapprochées et elles supportent des espèces d'arcades à jour sur lesquelles repose un plafond de bois (Rocca, 1887:162-163).

Les Mémoires se muent ainsi, par endroits, en récit de voyage dans lequel le narrateur transmet des expériences qui deviendront des topiques et que l'on retrouvera dans les narrations de voyage postérieures. De Gautier à Dumas, des voyageurs des années 20 à ceux des années 50 ou aux simples touristes des dernières années du siècle, on parlera du danger des chemins et des routes, des auberges et des contrebandiers. Les femmes andalouses et plus amplement les Espagnoles, acquièrent déjà, sous la plume de Rocca, des caractéristiques mauresques qu'elles n'abandonneront plus:

Les femmes du peuple s'asseyent encore, à la mauresque, sur des nattes de jonc circulaires, et, dans quelques couvens [sic] de l'Espagne où les anciennes coutumes sont transmises sans aucune altération, les religieuses sont encore dans l'habitude de s'asseoir comme les Turcs, sans savoir qu'elles tiennent cet usage des ennemis de la foi chrétienne. La mantilla, espèce de grand voile de laine que les femmes du peuple portent communément en Andalousie, et qui leur cache quelquefois toute la figure, excepté les yeux, a pour origine la pièce de drap dont les femmes s'enveloppent dans l'orient lorsqu'elles sortent. Les danses espagnoles, les diverses espèces de fandango sur-tout [sic], ressemblent beaucoup aux danses lascives de l'orient (Rocca, 1887:166).

L'Andalousie se pare déjà, aux yeux de ce jeune hussard, de toutes les vertus d'un paradis sur terre. Son émerveillement devant la fertilité de la région estompe le militaire pour ne laisser transparaître qu'un voyageur devant une réalité inconnue et presque féerique par son extraordinaire exubérance. Toute réalité quotidienne est contaminée par cette vision positive, et les chevaux, le pain, les olives, les coutumes, tout acquiert à ses yeux un aspect positif:

Le pain de l'Andalousie passe pour être le plus blanc et le plus exquis du monde entier, et les olives sont d'une grosseur surnaturelle. Le ciel de l'Andalousie est si serein et si pur, que 1 'on peut y dormir presque toute l'année en plein air; on voit pendant l'été, et quelquefois même pendant l'hiver, des hommes couchés sous des portiques. Il y a une foule d'individus peu riches, qui voyagent sans s'inquiéter de chercher chaque soir des logements; ils portent leurs vivres avec eux, ou bien ils achètent des alimens [sic] que des femmes préparent pour les passans [sic], sur des réchauds, à l'entrée des grandes villes, ou sur les places publiques. Les pauvres ne se demandent pas, comme dans les pays du nord, s'ils ont une maison pour demeurer, mais, s'ils ont un bon manteau, qui puisse les protéger en été contre le contact immédiat des rayons du soleil, et les préserver des pluies pendant l'hiver (Rocca, 1887: 160-161).

L'arrière-plan militaire se maintient dans ses comparaisons entre la campagne d'Egypte et celle de l'Espagne. Les passages descriptifs sur la manière de faire la guerre sont d'une longueur en accord avec la visée première du titre du récit, et l'esprit du soldat plane sur ces souvenirs de résistance inébranlable ${ }^{9}$. Son séjour à Ronda et le har-

${ }^{9}$ B. Mandopoulos met l'accent sur le répertoire d'images que les Romantiques tireront de l'Espagne, paradis perdu, et de ce «peuple fier de ses traditions », à partir des campagnes napoléoniennes: «À la charnière des 
cèlement continuel des paysans mérite une large place, et lui permet d'exprimer le caractère du peuple insoumis, qui allie patriotisme, fierté et générosité. Ayant failli mourir des suites d'une blessure reçue en combat, il restera plusieurs mois à Ronda, et il loue longuement le soin avec lequel il a été soigné par ses hôtes espagnols. La religiosité et la générosité du peuple sont mises en relief à travers le comportement de ses amphitryons, qui n'hésitent pas à le cacher de leurs compatriotes. La figure d'une femme âgée se détache avec un certain comique dans une scène qui dit en même temps le danger couru:

Une dame âgée, la mère de mon hôtesse, resta seule avec moi et elle se mit en prière ; elle tournait plus ou moins vite les grains de son chapelet, selon que les cris des combattans et les bruits des armes à feu annonçaient que le danger s'accroissait ou diminuait. Vers midi, la fusillade s'éloigna peu à peu, et cessa ensuite de se faire entendre. L'ennemi fut repoussé sur tous les points ; mes camarades vinrent, en descendant de cheval, me raconter le combat (Rocca, 1887:238).

C'est après cette pause qu'il entreprend son retour au pays, en passant encore une fois par Cordoue, et il le fait sans visiter donc ni Séville ni Grenade, celles qui deviendront les villes-phare des voyageurs qui suivront cette première vague de visiteurs militaires. Sa traversée de La Manche vers Madrid lui permet de signaler l'insécurité croissante et le malaise profond du peuple espagnol, auquel faisaient violence et les français et les quadrilles espagnoles: «Les habitans de la Manche, ainsi que ceux des autres provinces voisines, étaient exaspérés par tous ces genres de violences, et le nombre de nos ennemis s'accroissait chaque jour» (Rocca, 1887:243).

Certes, les Mémoires de John Rocca sont un récit de guerre: le titre l'atteste. Mais il est aussi certain que ce voyageur malgré lui peut être considéré comme un précurseur de la mode de la littérature de voyages, et notamment du voyage en Espagne, qui se développera sous toutes se formes au long du XIX siècle. Les militaires des campagnes napoléoniennes, agents et patients en même temps, ouvrent des fenêtres sur le futur, et à travers celle que Rocca a ouverte avec maestria, le lecteur perçoit déjà une Espagne telle que les voyageurs illustres qui le suivront vont la montrer aux lecteurs, et telle qu'elle va se perpétuer pendant des décennies. L'Espagne, ce lieu de mémoire, duquel se dégage l'image d'une civilisation différente et proche, permettra que d'autres voyageurs, quelque peu plus tard, réalisent leur propre périple.

Ces Mémoires montrent comment l'image littéraire de l'Espagne et de l'Andalousie commence à se forger très tôt. A côté des cruels tableaux de la Guerre de l'Indépendance, nous voyons se lever une image de l'Espagne qui va s'inscrire durablement dans l'imaginaire français. L'itinéraire référentiel guerrier se double d'une vision sur l'hétérogène, dans lequel le regard sur l'Autre s'avère fondamental. Ceci accorde à la narration, dont la qualité de l'écriture est indubitable, un statut double: elle est ancrée aussi bien dans le réel que dans le récit de voyage, lequel maintient de par sa nature même un lien

deux époques, l'hérö̈que résistance espagnole à l'entreprise napoléonienne n'est pas pour rien dans ce changement de perception. Quand la Grande Armée, porteuse des valeurs libérales de la Révolution Française, entre en Espagne en 1808, elle est convaincue de sa mission progressiste. Contre toute attente, elle se heurte à un peuple qui se bat farouchement contre un envahisseur aux prétentions coercitives » (2002: IV). 
avec la fiction. Les Mémoires sur la guerre des français en Espagne sont donc non seulement une simple description de récit de guerre, mais aussi une œuvre littéraire.

\section{BIBLIOGRAPHIE}

Aymes, Jean-René (1983): L'Espagne romantique, París, Éd. A. M. Métailié.

BENNASSAR, Bartolomé (1998): Le voyage en Espagne, Paris, R. Laffont.

GAUTIER, Théophile (1840): Voyage en Espagne, Paris, Gallimard 1981.

MANDOPOUlos, Béatrice (2002): «España es diferente », Espagne. Ombre et lumière . Textes réunis et présentés par B. Mandopoulos, París, Omnibus.

PageauX, Daniel-Henri (1995): «Recherche sur l'imagologie : de l'Histoire culturelle à la poétique», in Revista de Filología Francesa, 8, Servicio de publicaciones Univ. Complutense, Madrid, pp. 135-160.

RocCA, John (1814): Mémoires sur la guerre des Français en Espagne, Genève, Imprimerie JulesGuillaume Fick,1887.

SERrano, María del Mar (1993): «Viajes y viajeros por la España del siglo XIX», Cuadernos Críticos de Geografía Humana, n. ${ }^{\circ} 98$. 